Published in final edited form as:

Placenta. 2018 December ; 72-73: 20-27. doi:10.1016/j.placenta.2018.10.005.

\title{
Polymer Size Affects Biodistribution and Placental Accumulation of the Drug Delivery Biopolymer Elastin-Like Polypeptide in a Rodent Pregnancy Model
}

\author{
Marija Kuna1 ${ }^{1}$, Jamarius P. Waller ${ }^{2}$, Omar C. Logue ${ }^{2}$, and Gene L. Bidwell III ${ }^{1,2, *}$ \\ ${ }^{1}$ Department of Cell and Molecular Biology, University of Mississippi Medical Center, Jackson, \\ MS. \\ ${ }^{2}$ Department of Neurology, University of Mississippi Medical Center, Jackson, MS.
}

\begin{abstract}
Introduction.-Fusion of therapeutic agents to Elastin-like Polypeptide (ELP) is a novel drug delivery strategy for prevention of placental drug transfer. Previous studies have used a $60 \mathrm{kDa}$ ELP tag for this purpose. However, placental transfer of ELP may be size dependent. The goal of this study was to measure the effects of ELP polymer size on pharmacokinetics, biodistribution, and placental transfer of ELP.
\end{abstract}

Methods.-Three ELPs ranging from 25 to $86 \mathrm{kDa}$ ( 4.1 to $6.8 \mathrm{~nm}$ hydrodynamic radius) were fluorescently labeled and administered by i.v. bolus to pregnant Sprague Dawley rats on gestational day 14. Plasma levels were monitored for $4 \mathrm{~h}$, organ levels and placental transfer determined by ex vivo fluorescence imaging, and placental localization determined by confocal microscopy.

Results.-Increasing ELP size resulted in slower plasma clearance and increased deposition in all major maternal organs, except in the kidneys where an opposite effect was observed. Placental levels increased with an increase in size, while in the pups, little to no ELP was detected.

Discussion.-Pharmacokinetics and biodistribution of ELPs during pregnancy are size dependent, but all ELPs tested were too large to traverse the placental barrier. These studies verify that ELP fusion is a powerful method of modulating half-life and preventing placental transfer of cargo molecules. The tunable nature of the ELP sequence makes it ideal for drug delivery

\footnotetext{
"Correspondence: Gene L. Bidwell III, Department of Neurology, University of Mississippi Medical Center, 2500 North State Street, Jackson, MS 39216, USA, Tel +1 601984 6787, Fax +1 601984 6626, gbidwell@umc.Edu. AUTHOR CONTRIBUTIONS

G.L.B. conceived of and designed the research. G.L.B., J.P.W., O.C.L. and M.K. performed experiments. G.L.B. and M.K. analyzed the data. G.L.B. and M.K. interpreted the results of experiments. G.L.B. and M.K. prepared figures. G.L.B. and M.K. drafted the manuscript. G.L.B., J.P.W., O.C.L. and M.K. edited and revised the manuscript. All authors approved the final version of the manuscript. COMPETING INTERESTS

G.L.B. is owner of Leflore Technologies LLC, a private company working to commercialize ELP-based technologies in several disease areas. G.L.B. is author of pending patent application. M.K., J.P.W., and O.C.L. declare no conflict of interest.

Publisher's Disclaimer: This is a PDF file of an unedited manuscript that has been accepted for publication. As a service to our customers we are providing this early version of the manuscript. The manuscript will undergo copyediting, typesetting, and review of the resulting proof before it is published in its final citable form. Please note that during the production process errors may be discovered which could affect the content, and all legal disclaimers that apply to the journal pertain.
} 
applications during pregnancy, where it can be used to target drugs to the mother while preventing fetal drug exposure.

\section{Keywords}

elastin-like polypeptide; drug delivery system; molecular weight; pregnancy; placental accumulation

\section{INTRODUCTION}

In the U.S. alone, there were 3.98 million births reported in 2015. [1]. According to the FDA, at least $50 \%$ of these women took at least one medication during pregnancy. Yet many of those drugs had no clinical trials in pregnant women. Moreover, pregnancy is often an exclusion criteria for clinical trials due to possible deleterious effects to the fetus [2]. Drug development is additionally hindered because of risk aversion from the pharmaceutical industry and from regulatory bodies [3].

The current strategy for evaluating the safety of drugs that might be used during pregnancy requires initial reproductive toxicity testing in the preclinical and early clinical phase and post-approval monitoring. During preclinical development, studies of developmental and reproductive toxicology (DART) are typically done in mice, rats and rabbits [4].

Additionally, post-approval monitoring in pregnant subjects for drugs not restricted for use during pregnancy is often carried out as part of Phase IV studies. Still, actual patient data on effects of drugs during pregnancy is often scarce. Furthermore, direct drug development for adverse pregnancy-specific conditions is extremely limited. The need to administer drugs during pregnancy, especially drugs for treatment of pregnancy-related conditions remains high, underlying the need for development of tools to increase the safety of drugs administered during pregnancy.

A strategy recently explored by our lab involves the use of the drug delivery system elastinlike polypeptide (ELP) as a carrier for maternal drug sequestration. ELPs are genetically modified carriers originally developed by Urry and colleagues two decades ago [5,6]. ELPs consists of five amino acid repeats (pentamers), Val-Pro-Gly-Xaa-Gly, where Xaa is a guest residue and can be any amino acid except proline. ELPs can be easily modified to finetune their molecular weight by changing the number of pentamer repeats $[7,8]$, easily done through the use of recursive directional ligation [9], or to generate fusion proteins with therapeutic peptides, proteins [10-15], or reactive sites for small molecule drug conjugation [16-18]. ELP fusion to therapeutic peptides and small proteins increases their stability, and they can increase solubility and reduce off-target toxicity of small molecule drugs. They can be engineered to form nanoparticles for drug delivery [19-21], to form hydrogels for controlled drug release [22,23], or for targeted drug delivery using externally applied focused hyperthermia [24-26].

Many small molecule drugs can easily cross the placenta, and placental transfer depends on their hydrophobicity and charge. Large molecules such as proteins are often prevented from passive crossing by the placental barrier. Some proteins, such as immunoglobulins, are transported across the placental barrier via receptors, FcRn, expressed on 
syncytiotrophoblasts [27-29]. Transferrin also has receptors on trophoblasts and is actively transported across placental barrier [30,31]. Some high molecular weight (MW) drug carriers have been investigated for placental transfer with highly variable results. Some types of nanoparticles readily cross the placenta and some do not [32-34].Taken as a whole, these studies show that placental transfer isn't restricted by merely the size of macromolecular drug carriers.

Previous work has been done to look at placental transfer of ELPs by our group. Using a rat pregnancy model, the placental transfer of an intermediately sized ELP (61 kDa, similar to the size used for many previous ELP drug delivery studies [2,18,24,25,35,36] was determined after two different routes of administration, a single, bolus intravenous injection and a continuous intraperitoneal administration for five days. On gestational day 14 (GD14), little to no ELP crossed placental barrier after a high-dose bolus injection. Furthermore, the maternal sequestration held even after five days of continuous infusion or after modifying the ELP to include a cell penetrating peptide [2]. This previous work suggests that this delivery system could be utilized for prevention of fetal drug exposure.

Recently, our lab has developed a library of ELPs with varying MW. The MW has strong effects on the polymer's hydrodynamic radius, pharmacokinetics, and biodistribution. We measured the physical and biological properties of these molecules, including their hydrodynamic radius, in vitro stability, pharmacokinetics and biodistribution [37]. The smallest ELP tested included 63 VPGxG repeats. This protein had a predicted molecular weight of $25.2 \mathrm{kDa}$, and dynamic light scattering revealed its hydrodynamic radius to be $4.17 \pm 0.06 \mathrm{~nm}$. The range of proteins tested extended up to an ELP with over $200 \mathrm{VPGxG}$ repeats, and molecular size increased with increasing repeat number until an asymptote was reached at a hydrodynamic radius of approximately $7 \mathrm{~nm}$. Increasing the ELP size resulted in increased plasma half-life and altered deposition in various tissues, including a change in intra-renal localization from exclusively cortical for the smallest ELPs to a mixture of cortical and medullary for the larger ELPs. Considering that pregnancy results in multisystem changes that could alter pharmacokinetics and tissue accumulation of these macromolecules, we wanted to determine how molecular weight impacts pharmacokinetics and biodistribution of ELPs during pregnancy. Furthermore, the body of macromolecule and nanoparticle literature shows that size alone is not a good predictor of placental transfer, so we endeavored to determine if ELP size affected its placental transfer and placental deposition. We hypothesized that, due to an increase in ELP plasma half-life combined with the large blood pool in the placenta, accumulation of ELP carriers in maternal organs, especially in the placenta, would be increased with an increase in ELP MW. Three ELP sizes inclusive of the entire range of our previously published size library were chosen for this study. The relevant molecular weights, transition temperatures, and hydrodynamic radii for the proteins used in this study are shown in Table 1.

\section{METHODS}

\section{Synthesis of ELP Expression Constructs by Recursive Directional Ligation.}

We generated a library of ELP constructs using recursive directional ligation as described by Kuna et al. [37]. 


\section{Purification of ELP Proteins.}

pET25b+ vectors encoding ELP proteins were transformed into E. coli BLR (DE3). All proteins were purified by inverse transition cycling, as described previously [38,11,37].

\section{Fluorescent Labeling of ELP Proteins.}

Each ELP protein was labeled on its $\mathrm{N}$-terminal cysteine residue using a maleimide conjugate of rhodamine as described by Kuna et al. [37] using a method modified from [38].

\section{Pharmacokinetic Studies.}

Animal studies were approved by the Animal Care and Use Committee of the University of Mississippi Medical Center and conducted according to the guidelines of the Guide for the Care and Use of Laboratory Animals [39]. For pharmacokinetic and biodistribution experiments, three ELPs ranging in MW from $25 \mathrm{kDa}$ to $86 \mathrm{kDa}$ were used. Sprague Dawley timed pregnant rats on GD14 (Charles River) were anesthetized with isoflurane (1-3\%, to effect) and injected with rhodamine-labeled polypeptides $(1.5 \mu \mathrm{mol} / \mathrm{kg})$ by intravenous injection into the femoral vein. Blood was sampled by tail prick at various time points for 4 hours, collected in Greiner Bio-One MiniCollect capillary blood collection tubes (Greiner Bio-One), and plasma was collected after centrifugation. Plasma samples were analyzed for concentration of the polypeptides using quantitative fluorescence analysis as described by Kuna et al. [37].

\section{Biodistribution, Placental and Kidney Localization of ELPs.}

For tissue biodistribution studies, the same animals from the pharmacokinetic study were euthanized while still under anesthesia. Their urine, placenta and pups, and organs were collected for whole organ fluorescence biodistribution analysis $(\mathrm{n}=4$ rats per agent). Major organs and placentae and their associated pups ( $\mathrm{n}>5$ per animal) were imaged ex vivo using an IVIS Spectrum. Total organ fluorescence was quantified and fit to standard curves of the appropriate ELP as described in [37] to correct for any differences in labeling levels among polypeptides.

One amniotic sac, containing a pup and its placenta, was removed from each animal and kept intact. These feto-amnio-placental units were then embedded in freezing medium (Tissue-Plus O.C.T Compound) and flash frozen in isopentane on dry ice as described by McGowan [40]. Placentae and pups were cut into $20 \mu \mathrm{m}$ sections with a cryostat. Kidneys were also embedded, flash frozen and cut into $14 \mu \mathrm{m}$ sections. Slides were equilibrated to room temperature, and unprocessed tissue sections were imaged by confocal microscopy image stitching using a $561 \mathrm{~nm}$ laser and a 10x magnification objective. The same imaging settings were maintained for all samples. Brightness was adjusted equally among all groups to allow for better visualization while still maintaining quantitative differences in fluorescence levels.

\section{Urine Analysis.}

Urine samples collected prior to euthanasia four hours after protein injection were analyzed by quantitative fluorescence analysis. The fluorescence intensity of $2 \mu \mathrm{l}$ of urine was 
measured in a plate reader before and after trichloroacetic acid (TCA) precipitation as described in [37]. Post-precipitation levels were corrected for dilution and compared to preprecipitation fluorescence to calculate percentage of free dye. Urine samples were analyzed for creatinine levels using QuantiChrom Creatinine Assay Kit (DICT-500, BioAssay Systems) per Manufacturer's instructions. Urine samples were also analyzed by SDS-PAGE. $10 \mu \mathrm{l}$ of each urine sample was prepared with Bolt LDS sample buffer 4x (Novex, Thermo Fisher Scientific), analyzed on a Bolt 4-12\% Bis-tris Plus gel, and visualized by direct fluorescence imaging using an IVIS Spectrum (PerkinElmer) using 535-nm excitation and 580-nm emission filters and small binning, followed by Coomassie Brilliant Blue staining.

\section{Statistical Analysis.}

Plasma ELP levels were assessed with a two-way repeat measures ANOVA for factors of polypeptide treatment and time with a post hoc Tukey's multiple comparison. Organ biodistribution was assessed with a two-way ANOVA for factors of polypeptide treatment and organ type with post hoc Tukey's multiple comparison. The relationship between MW and organ levels was determined by Pearson correlation coefficient. Urine free dye and rhodamine levels were assessed by a one-way ANOVA for differences in polypeptide treatment with post hoc Tukey's multiple comparison. In all analyses, a p value of $<0.05$ was considered statistically significant. All statistical analysis and curve fitting was done using GraphPad Prism (Version 7.04).

\section{Data availability.}

Experimental data are available from the authors upon request.

\section{RESULTS}

\section{Plasma Clearance of ELP Proteins.}

A pharmacokinetic study was conducted in SD timed pregnant rats on GD14 to determine the effects of MW on plasma clearance of ELPs. This time point was chosen as it represents the first day of the third week of the rodent gestation, a time at which we are utilizing ELPs to deliver therapeutics in our preeclampsia drug development studies [36]. Three ELPs with MWs of 25, 50, and $86 \mathrm{kDa}$ were administered by bolus IV injection. Based on direct fluorescence measurement of plasma (Fig. 1A), an increase in MW of these proteins resulted in slower plasma clearance. All of the proteins used had the highest plasma concentration immediately after bolus i.v. injection at 5 min time point. ELP-63 plasma levels were lower than ELP-127 and ELP-223 at all time points tested, and ELP-127 plasma levels were lower than ELP-223 plasma levels at the $2 \mathrm{~h}$ and $2.5 \mathrm{~h}$ time points. At the four hour time point, the smallest protein ELP-63 (25 kDa) levels were lowest at 0.35 $\pm 0.06 \mu \mathrm{M}$, while ELP-127 (50 $\mathrm{kDa}$ ) was $2.09 \pm 0.45 \mu \mathrm{M}$, and the largest protein ELP-223 (86 kDa) was $5.66 \pm 1.35 \mu \mathrm{M}$. While four hours of data were not enough to obtain reliable pharmacokinetic fits, these trends in plasma clearance are consistent with our observations for these proteins in nonpregnant mice, in which we measured 48 hours of plasma clearance data. However, extended plasma clearance time points were not measured in this study because the animals were euthanized to retrieve tissues for biodistribution analysis. Further analysis by TCA 
precipitation revealed that the fluorescence present in the plasma was not free fluorophore (Fig. 1B). None of the proteins tested had more than $6 \%$ free dye in the plasma.

\section{Biodistribution of ELP Proteins.}

Four hours after the injection, a biodistribution study was carried out to determine changes in tissue accumulation of ELPs with varying MW. After euthanasia, major organs, including placentae and pups, were removed to quantify ELP tissue levels. Representative images of major organs, placentae, and pups for each of the treatment groups are shown in Fig. 2A and B. The high renal accumulation of all ELPs tested is apparent in Fig. 2A. Fig. 2B shows that there is also strong ELP signal in the placenta, especially for ELP-127 and ELP-223, but there is no detectable ELP in the pups. The data from these images was quantified relative to standard curves of the injected proteins as shown in Fig. 2C. The ELP proteins used in the study accumulated strongly in the kidneys regardless of their MW, followed by the liver and placenta. Tissue levels were dependent on ELP MW, with all organs showing trends for increased ELP deposition with increasing MW except the kidney, in which ELP levels significantly decreased with increasing MW (Two-way ANOVA with post hoc Tukey's multiple comparison, $\mathrm{F}(2,72)=3.56, \mathrm{p}=0.0335)$. The relationship between $\mathrm{MW}$ and organ levels was determined by a Pearson correlation coefficient, and the results of the analysis are reported in Table 2. Brain, lungs, liver, heart and placenta ELP levels had a positive correlation with ELP MW, with Pearson correlation coefficients $r>0.9$, while the kidneys levels had a negative correlation with MW with an $r=-0.9$. For all organs, the $\mathrm{R}^{2}$ was $>0.8$, and while a trend for correlation was apparent, only the brain correlation had a $\mathrm{p}$ value $<0.05$ (Fig 2D).

\section{Urine Analysis.}

Urine was collected from the animals at the time of organ harvest, four hours after bolus i.v. injection of fluorescently labeled proteins. Urine fluorescence was fit to a standard curve, corrected for rhodamine and creatinine concentration, and assessed by one-way ANOVA with post hoc Tukey's multiple comparison $(\mathrm{F}(2,9)=5.706, \mathrm{p}=0.0251)$. Rhodamine levels in the urine corrected by creatinine were significantly different between ELP-127 and ELP-223, as assessed by one-way ANOVA with post hoc Tukey's multiple comparison (Fig. $3 \mathrm{~A})$. Analysis of fluorescence before and after TCA precipitation revealed that the urine contained 78, 72 and $69 \%$ free fluorophore for ELP-63, ELP-127, and ELP-223 (Fig 3B), respectively. These levels were not significantly different (one-way ANOVA with post hoc Tukey's multiple comparison $(\mathrm{F}(2,9)=2.994, \mathrm{p}=0.1007)$. There was no correlation between ELP MW and either urine fluorescence levels or percent free dye (Pearson's correlation coefficient for urine $\%$ free dye and rhodamine concentration corrected by creatinine had a negative value of -0.9594 and -0.8121 , respectively. $R^{2}$ values were 0.9205 and 0.6595 , and $\mathrm{p}=0.1819$ and 0.3967 , respectively). Gel electrophoresis with direct fluorescence detection revealed that no intact ELP was present in the urine (Fig 3C). A major band was present at the bottom of the gel, representing free rhodamine, and a less intense band was apparent just above the free rhodamine band likely indicating dye bound to an amino acid or very short peptide fragment. These results indicate that no ELP passes intact to the urine, and they are consistent with our previous findings in mice that ELPs are strongly reabsorbed in the kidneys by the tubular protein reuptake system. A Coomassie stain revealed a similar 
banding pattern for all rats, with no bands correlating to full length ELP and similar loading levels among lanes (though the levels of an $\sim 60 \mathrm{kDa}$ protein, likely albumin, did vary from rat to rat).

\section{Placental and Kidney Localization of ELP Proteins.}

In addition to whole organ ex vivo imaging, we also imaged sections of intact feto-amnioplacental units (Fig. 4A) and kidneys (Fig. 5) by confocal microscopy. There was an increase in placental levels with an increase in MW as shown in Fig. 4A. All of the ELPs had high accumulation at the chorionic plate, with the largest ELP-223 having the highest placental level and broad distribution throughout the labrynth and junctional zone. No ELP signal was detected in the fetus.

Kidney sections revealed similar localization as was observed in our previous mouse study [37] with the smallest ELP-63 (25 kDa) being exclusively cortical, and largest ELP-223 (86 $\mathrm{kDa}$ ) having a more medullary signal (Figure 5A, images collected with identical parameters to demonstrate actual protein levels). Magnification revealed complete lack of medullary deposition of ELP-63, however, ELP-223 accumulated in distinct medullary structures (Figure 5B, arrow, image intensity adjusted individually to optimize visibility of protein localization).

\section{DISCUSSION}

Considering the widespread need for drug development to treat pregnant women and pregnancy-related conditions, we seek to develop tools to increase the safety of drugs administered during pregnancy. One of such tools is ELP, a bioengineered polymer used originally for tumor targeted delivery [24-26]. In recent years these carriers have been investigated for a multitude of other applications, from their use as slow release depots $[23,41,42]$, for ocular drug delivery [43,44], for treatment of kidney disease [35,45], and to treat preeclampsia [36]. These molecules are attractive for delivery during pregnancy because of the recent observation that they do not cross the placental barrier [2,36].

Previous studies have investigated placental transfer of other large drug carriers including nanoparticles. Some, including liposomes, irrespective of size, charge or lipid composition, do not cross the placenta in a human placental perfusion model [46]. Also, PEGylated gold nanoparticles, $10-30 \mathrm{~nm}$ in diameter, were not detected in fetal outflow in a human placental perfusion model [47]. However, other gold nanoparticles, $13 \mathrm{~nm}$ in diameter with different surface modifications, all crossed the placental barrier prior to embryonic day 11.5 in a murine pregnancy model [32]. Polystyrene beads with diameters up to $240 \mathrm{~nm}$ and PAMAM dendrimers were able to cross the placental barrier in the ex vivo human placental perfusion model, though with varying efficiency [33,34]. These results demonstrate that placental transfer can be difficult to predict a priori even for nanoparticles that are much larger than our soluble ELP proteins and lead us to undertake the current study examining the size dependence of ELP placental transfer. Other studies have systematically investigated how MW influences pharmacokinetics and tumor accumulation of ELP. Ryu et al. investigated the influence of MW on ELP tumor accumulation and found that tumor accumulation increased with an increase in MW [48]. Recent work by our lab has shown that increasing 
the ELP size resulted in increased plasma half-life and altered deposition in various tissues [37]. As pregnancy involves a myriad of physiological changes including significant volume expansion and vasodilation [49], which can ultimately alter pharmacokinetics and tissue accumulation of these macromolecules, we wanted to determine how molecular weight impacts pharmacokinetics and biodistribution of ELPs during pregnancy and, specifically, their placental transfer and placental deposition.

Our first goal was to determine if pregnancy alters the pharmacokinetics of these proteins in relationship with MW. We found that the larger ELPs had higher plasma levels and slower plasma clearance relative to the smaller ELPs (Fig. 1A), as expected based on the literature [50] and consistent with our previous results in non-pregnant rodents [51]. ELPs accumulated most predominantly in the kidneys, followed by liver, placenta, and brain (Fig. 2C). An increase in MW resulted in a decrease in kidney levels, which was consistent with our data previously collected in mice by quantitative fluorescence histology [37]. The intrarenal localization was similar in both species, and the trend for decreased cortical and increased medullary protein deposition was observed in both studies. Unlike the kidneys, in all the other organs including the placenta, an increase in MW resulted in an increase in tissue levels (Fig. 2D and Table 2), possibly indicating that the larger sized proteins undergo slower clearance after extravasation in peripheral tissues.

Examination of the urine from these animals did not reveal significant differences in the amount of free dye between the smallest and intermediate sized ELPs, though less free dye was in the urine of rats treated with the largest ELP (Fig. 3). This is consistent with the decrease in kidney levels and increase in other organ levels as ELP size increases, reflecting the fact that more ELP was present elsewhere in the maternal body as the polymer size is increased. This could also indicate that larger ELPs aren't as easily filtered, which is consistent with our previously published data in mice where ELP-223 formed aggregates in the glomerulus and in medullary structures. In the urine, the majority of the signal was from free rhodamine, but $20-30 \%$ of the fluorophore was still bound to a small but TCAprecipitatable protein component, likely a short peptide degradation product. These results indicate that no ELP passes intact to the urine. The intra-renal ELP localization in pregnant rats was consistent with our previous data in nonpregnant mice showing that with an increase in size, ELP accumulation in the cortex decreases and ELP levels in medulla increase.

Further analysis of placental sections revealed that ELPs regardless of their MW accumulated most prominently in the chorionic plate, consistent with previously published data, and were also distributed diffusely within the labyrinth and junctional zone [51]. The diffuse labyrinth and junctional zone accumulation increased with increasing ELP size, likely reflecting the increased amount of ELP in the blood pool as size increased. There is no know receptor for ELP, it is not known to bind to the neonatal $\mathrm{Fc}$ receptor, and there is likely no means for active transport of ELP across the placental barrier. Therefore, it is likely that ELPs, even those as small as the $25 \mathrm{kDa}$ construct tested in this study, are excluded from placental transfer by the placental size barrier. In combination with ex vivo organ levels, these data confirm that ELP could be utilized as a drug delivery system to prevent fetal drug exposure. Ex vivo organ analysis revealed an increase in placenta levels when compared to 
pup levels with an increase in MW, which indicated that higher MW ELPs would be a better carrier for placental delivery. This is particularly beneficial as these molecules also have a slower plasma clearance and would have a prolonged chance of reaching their target. However, other details need to be taken into account when developing an ELP-therapeutic fusion for use during pregnancy. First, it must be determined if therapeutic release from the ELP carrier is necessary for drug action. This is likely to be the case for many small molecule drug fusions. If so, drug release must be confirmed at the desired target site, and the potential still exists for drug to cross the placental barrier after cleavage from the ELP carrier, so this must be carefully monitored for each individual ELP fusion application. In many of the therapeutics our lab is developing for treatment of preeclampsia, the ELP-fusion protein is active as a chimera, resulting in a novel type of biologic.

In many cases, these ELP-fusion protein biologics target proteins that are circulating in plasma or receptors present on endothelial cells. Thus no release of the therapeutic from the ELP carrier is necessary and concerns over placental transfer of the therapeutic agent are mitigated.

\section{Acknowledgments}

FUNDING

This work was supported by NIH NHLBI Grant R01HL121527 (G.L.B.).

\section{REFERENCES}

[1]. Martin JA, Hamilton BE, Osterman MJ, Driscoll AK, Mathews TJ, Births: final data for 2015, National Vital Statistics Reports. 66 (2017).

[2]. George EM, Liu H, Robinson GG, Bidwell GL, A polypeptide drug carrier for maternal delivery and prevention of fetal exposure, Journal of Drug Targeting. 22 (2014) 935-947. doi: 10.3109/1061186X.2014.950666. [PubMed: 25148609]

[3]. Ilekis JV, Tsilou E, Fisher S, Abrahams VM, Soares MJ, Cross JC, Zamudio S, Illsley NP, Myatt L, Colvis C, Placental origins of adverse pregnancy outcomes: potential molecular targets: an Executive Workshop Summary of the Eunice Kennedy Shriver National Institute of Child Health and Human Development, American Journal of Obstetrics \& Gynecology. 215 (2016) S1-S46. [PubMed: 26972897]

[4]. Frederiksen MC, The drug development process and the pregnant woman, J Midwifery Womens Health. 47 (2002) 422-425. [PubMed: 12484663]

[5]. Urry DW, Free energy transduction in polypeptides and proteins based on inverse temperature transitions, Progress in Biophysics and Molecular Biology. 57 (1992) 23-57. doi: 10.1016/0079-6107(92)90003-O. [PubMed: 1549698]

[6]. Urry DW, Physical Chemistry of Biological Free Energy Transduction As Demonstrated by Elastic Protein-Based Polymers, J. Phys. Chem. B. 101 (1997) 11007-11028. doi:10.1021/jp972167t.

[7]. Chilkoti A, Dreher MR, Meyer DE, Design of thermally responsive, recombinant polypeptide carriers for targeted drug delivery, Advanced Drug Delivery Reviews. 54 (2002) 1093-1111. [PubMed: 12384309]

[8]. Meyer DE, Chilkoti A, Genetically encoded synthesis of protein-based polymers with precisely specified molecular weight and sequence by recursive directional ligation: examples from the elastin-like polypeptide system, Biomacromolecules. 3 (2002) 357-367. [PubMed: 11888323]

[9]. McDaniel JR, MacKay JA, Quiroz FG, Chilkoti A, Recursive directional ligation by plasmid reconstruction allows rapid and seamless cloning of oligomeric genes, Biomacromolecules. 11 (2010) 944-952. [PubMed: 20184309] 
[10]. Meyer DE, Chilkoti A, Purification of recombinant proteins by fusion with thermally-responsive polypeptides, Nat. Biotechnol. 17 (1999) 1112-1115. doi: 10.1038/15100. [PubMed: 10545920]

[11]. George EM, Liu H, Robinson GG, Mahdi F, Perkins E, Bidwell GL, Growth factor purification and delivery systems (PADS) for therapeutic angiogenesis, Vascular Cell. 7 (2015) 1. doi: 10.1186/s13221-014-0026-3. [PubMed: 25653833]

[12]. Bidwell GL, Perkins E, Raucher D, A thermally targeted c-Myc inhibitory polypeptide inhibits breast tumor growth, Cancer Lett. 319 (2012) 136-143. doi:10.1016/j.canlet.2011.12.042. [PubMed: 22261328]

[13]. Patel J, Zhu H, Menassa R, Gyenis L, Richman A, Brandle J, Elastin-like polypeptide fusions enhance the accumulation of recombinant proteins in tobacco leaves, Transgenic Res. 16 (2007) 239-249. doi:10.1007/s11248-006-9026-2. [PubMed: 17106768]

[14]. Massodi I, Moktan S, Rawat A, Bidwell GL, Raucher D, Inhibition of ovarian cancer cell proliferation by a cell cycle inhibitory peptide fused to a thermally responsive polypeptide carrier, Int. J. Cancer. 126 (2010) 533-544. doi:10.1002/ijc.24725. [PubMed: 19588502]

[15]. Amiram M, Luginbuhl KM, Li X, Feinglos MN, Chilkoti A, Injectable proteaseoperated depots of glucagon-like peptide-1 provide extended and tunable glucose control, Proc. Natl. Acad. Sci. U.S.A. 110 (2013) 2792-2797. doi:10.1073/pnas.1214518110. [PubMed: 23359691]

[16]. Dreher MR, Raucher D, Balu N, Michael Colvin O, Ludeman SM, Chilkoti A, Evaluation of an elastin-like polypeptide-doxorubicin conjugate for cancer therapy, J Control Release. 91 (2003) 31-43. [PubMed: 12932635]

[17]. Bidwell GL, Davis AN, Fokt I, Priebe W, Raucher D, A thermally targeted elastin-like polypeptide-doxorubicin conjugate overcomes drug resistance, Invest New Drugs. 25 (2007) 313-326. doi:10.1007/s10637-007-9053-8. [PubMed: 17483874]

[18]. Moktan S, Perkins E, Kratz F, Raucher D, Thermal targeting of an acid-sensitive doxorubicin conjugate of elastin-like polypeptide enhances the therapeutic efficacy compared with the parent compound in vivo, Molecular Cancer Therapeutics. 11 (2012) 1547-1556. [PubMed: 22532601]

[19]. Yeboah A, Cohen RI, Faulknor R, Schloss R, Yarmush ML, Berthiaume F, The development and characterization of SDF1a-elastin-like-peptide nanoparticles for wound healing, J Control Release. 232 (2016) 238-247. doi:10.1016/j.jconrel.2016.04.020. [PubMed: 27094603]

[20]. Dhandhukia JP, Shi P, Peddi S, Li Z, Aluri S, Ju Y, Brill D, Wang W, Janib SM, Lin Y-A, Liu S, Cui H, MacKay JA, Bifunctional Elastin-like Polypeptide Nanoparticles Bind Rapamycin and Integrins and Suppress Tumor Growth in Vivo, Bioconjug. Chem. (2017). doi:10.1021/ acs.bioconjchem.7b00469.

[21]. Monfort DA, Koria P, Recombinant elastin-based nanoparticles for targeted gene therapy, Gene Ther. 24 (2017) 610-620. doi:10.1038/gt.2017.54. [PubMed: 28681841]

[22]. Zhang Y-N, Avery RK, Vallmajo-Martin Q, Assmann A, Vegh A, Memic A, Olsen BD, Annabi N, Khademhosseini A, A Highly Elastic and Rapidly Crosslinkable Elastin-Like PolypeptideBased Hydrogel for Biomedical Applications, Adv Funct Mater. 25 (2015) 4814-4826. doi : 10.1002/adfm.201501489. [PubMed: 26523134]

[23]. Schaal JL, Li X, Mastria E, Bhattacharyya J, Zalutsky MR, Chilkoti A, Liu W, Injectable polypeptide micelles that form radiation crosslinked hydrogels in situ for intratumoral radiotherapy, J Control Release. 228 (2016) 58-66. doi:10.1016/j.jconrel.2016.02.040. [PubMed: 26928529]

[24]. Meyer DE, Kong GA, Dewhirst MW, Zalutsky MR, Chilkoti A, Targeting a genetically engineered elastin-like polypeptide to solid tumors by local hyperthermia, Cancer Res. 61 (2001) 1548-1554. [PubMed: 11245464]

[25]. Bidwell III GL, Perkins E, Hughes J, Khan M, James JR, Raucher D, Thermally targeted delivery of a c-Myc inhibitory polypeptide inhibits tumor progression and extends survival in a rat glioma model, PloS One. 8 (2013) e55104. [PubMed: 23372821]

[26]. Bidwell III GL, Perkins E, Raucher D, A thermally targeted c-Myc inhibitory polypeptide inhibits breast tumor growth, Cancer Letters. 319 (2012) 136-143. doi: 10.1016/j.canlet. 2011.12.042. [PubMed: 22261328] 
[27]. Story CM, Mikulska JE, Simister NE, A major histocompatibility complex class I-like Fc receptor cloned from human placenta: possible role in transfer of immunoglobulin $\mathrm{G}$ from mother to fetus, J. Exp. Med. 180 (1994) 2377-2381. [PubMed: 7964511]

[28]. Leach JL, Sedmak DD, Osborne JM, Rahill B, Lairmore MD, Anderson CL, Isolation from human placenta of the IgG transporter, FcRn, and localization to the syncytiotrophoblast: implications for maternal-fetal antibody transport., The Journal of Immunology. 157 (1996) 3317-3322. [PubMed: 8871627]

[29]. Firan M, Bawdon R, Radu C, Ober RJ, Eaken D, Antohe F, Ghetie V, Ward ES, The MHC class I-related receptor, FcRn, plays an essential role in the maternofetal transfer of gamma-globulin in humans, Int. Immunol. 13 (2001) 993-1002. [PubMed: 11470769]

[30]. Galbraith GM, Galbraith RM, Faulk WP, Immunological studies of transferrin and transferrin receptors of human placental trophoblast, Placenta. 1 (1980) 33-46. [PubMed: 6255455]

[31]. McArdle HJ, Morgan EH, Transferrin and iron movements in the rat conceptus during gestation, J.Reprod. Fertil. 66 (1982) 529-536. [PubMed: 7175808]

[32]. Yang H, Sun C, Fan Z, Tian X, Yan L, Du L, Liu Y, Chen C, Liang X, Anderson GJ, Keelan JA, Zhao Y, Nie G, Effects of gestational age and surface modification on materno-fetal transfer of nanoparticles in murine pregnancy, Sci Rep. 2 (2012). doi: 10.1038/srep00847.

[33]. Wick P, Malek A, Manser P, Meili D, Maeder-Althaus X, Diener L, Diener P-A, Zisch A, Krug HF, von Mandach U, Barrier Capacity of Human Placenta for Nanosized Materials, Environ Health Perspect. 118 (2010) 432-436. doi:10.1289/ehp.0901200. [PubMed: 20064770]

[34]. Menjoge AR, Rinderknecht AL, Navath RS, Faridnia M, Kim CJ, Romero R, Miller RK, Kannan RM, Transfer of PAMAM dendrimers across human placenta: prospects of its use as drug carrier during pregnancy, J Control Release. 150 (2011) 326-338. doi:10.1016/j.jconrel.2010.11.023. [PubMed: 21129423]

[35]. Meyer DE, Shin BC, Kong GA, Dewhirst MW, Chilkoti A, Drug targeting using thermally responsive polymers and local hyperthermia, J Control Release. 74 (2001) 213-224. [PubMed: 11489497]

[36]. Chade AR, Tullos NA, Harvey TW, Mahdi F, Bidwell GL, Renal Therapeutic Angiogenesis Using a Bioengineered Polymer-Stabilized Vascular Endothelial Growth Factor Construct, J. Am. Soc. Nephrol. 27 (2016) 1741-1752. doi:10.1681/ASN.2015040346. [PubMed: 26541349]

[37]. Logue OC, Mahdi F, Chapman H, George EM, Bidwell GL, A Maternally Sequestered, Biopolymer-Stabilized Vascular Endothelial Growth Factor (VEGF) Chimera for Treatment of Preeclampsia, Journal of the American Heart Association. 6 (2017) e007216. doi: 10.1161/ JAHA.117.007216. [PubMed: 29629873]

[38]. Kuna M, Mahdi F, Chade AR, Bidwell GL, Molecular Size Modulates Pharmacokinetics, Biodistribution, and Renal Deposition of the Drug Delivery Biopolymer Elastin-like Polypeptide, Sci Rep. 8 (2018) 7923. doi:10.1038/s41598-018-24897-9. [PubMed: 29784932]

[39]. Bidwell GL, Raucher D, Application of thermally responsive polypeptides directed against cMyc transcriptional function for cancer therapy, Molecular Cancer Therapeutics. 4 (2005) 10761085. [PubMed: 16020665]

[40]. National Research Council, D. on E. and L. Studies, I. for L.A. Research, C. for the U. of the G. for the C. and U. of L. Animals, Guide for the Care and Use of Laboratory Animals: Eighth Edition, National Academies Press, 2010.

[41]. McGowan JWD, Bidwell III GL, The Use of Ex Vivo Whole-organ Imaging and Quantitative Tissue Histology to Determine the Bio-distribution of Fluorescently Labeled Molecules, J Vis Exp. (2016). doi:10.3791/54987.

[42]. Amiram M, Luginbuhl KM, Li X, Feinglos MN, Chilkoti A, A depot-forming glucagon-like peptide-1 fusion protein reduces blood glucose for five days with a single injection, J Control Release. 172 (2013) 144-151. doi:10.1016/j.jconrel.2013.07.021. [PubMed: 23928357]

[43]. Mukerji R, Schaal J, Li X, Bhattacharyya J, Asai D, Zalutsky MR, Chilkoti A, Liu W, Spatiotemporally photoradiation-controlled intratumoral depot for combination of brachytherapy and photodynamic therapy for solid tumor, Biomaterials. 79 (2016) 79-87. doi: 10.1016/ j.biomaterials.2015.11.064. [PubMed: 26702586] 
[44]. Wang W, Jashnani A, Aluri SR, Gustafson JA, Hsueh P-Y, Yarber F, McKown RL, Laurie GW, Hamm-Alvarez SF, MacKay JA, A thermo-responsive protein treatment for dry eyes, J Control Release. 199 (2015) 156-167. doi:10.1016/j.jconrel.2014.11.016. [PubMed: 25481446]

[45]. George EM, Mahdi F, Logue OC, Robinson GG, Bidwell GL, Corneal Penetrating Elastin-Like Polypeptide Carriers, J Ocul Pharmacol Ther. 32 (2016) 163-171. doi:10.1089/jop.2015.0082. [PubMed: 26672799]

[46]. Chade AR, Williams ML, Guise E, Vincent LJ, Harvey TW, Kuna M, Mahdi F, Bidwell GL, Bioploymer-delivered VEGF for Therapeutic Angiogenesis in Experimental Renovascular Disease: Targeting the Kidney Via Systemic Administration, Kidney International. In press (2017).

[47]. Bajoria R, Sooranna SR, Liposome as a drug carrier system: Prospects for safer prescribing during pregnancy: A review, Placenta. 19 (1998) 265-287.

[48]. Myllynen PK, Loughran MJ, Howard CV, Sormunen R, Walsh AA, Vähakängas KH, Kinetics of gold nanoparticles in the human placenta, Reproductive Toxicology. 26 (2008) 130-137. [PubMed: 18638543]

[49]. Ryu JS, Raucher D, Elastin-like polypeptides: the influence of its molecular weight on local hyperthermia-induced tumor accumulation, European Journal of Pharmaceutics and Biopharmaceutics. 88 (2014) 382-389. [PubMed: 24866938]

[50]. Cheung KL, Lafayette RA, Renal physiology of pregnancy, Adv Chronic Kidney Dis. 20 (2013) 209-214. doi:10.1053/j.ackd.2013.01.012. [PubMed: 23928384]

[51]. Dreher MR, Liu W, Michelich CR, Dewhirst MW, Yuan F, Chilkoti A, Tumor vascular permeability, accumulation, and penetration of macromolecular drug carriers, Journal of the National Cancer Institute. 98 (2006) 335-344. [PubMed: 16507830]

[52]. Bidwell GL, George EM, Maternally sequestered therapeutic polypeptides - a new approach for the management of preeclampsia, Front Pharmacol. 5 (2014) 201. doi: 10.3389/fphar. 2014.00201. [PubMed: 25249978]

Placenta. Author manuscript; available in PMC 2019 December 01. 
- Increase in ELP size results in slower plasma clearance in rodent pregnancy model

- $\quad$ Kidney ELP levels decrease with an increase in ELP size

- Increase in ELP size increases their placental accumulation

- There is no placental transfer regardless of the change in ELP size 
A

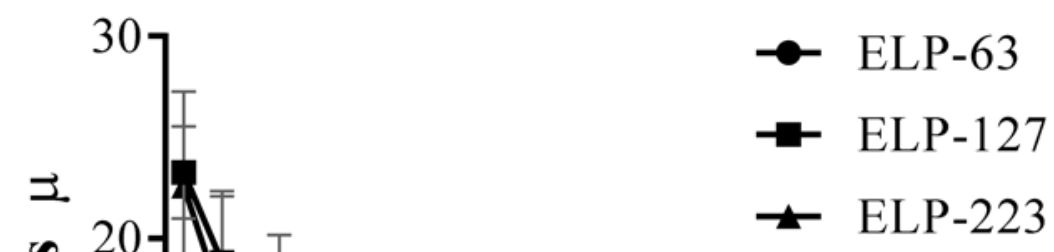

B

Time (h)

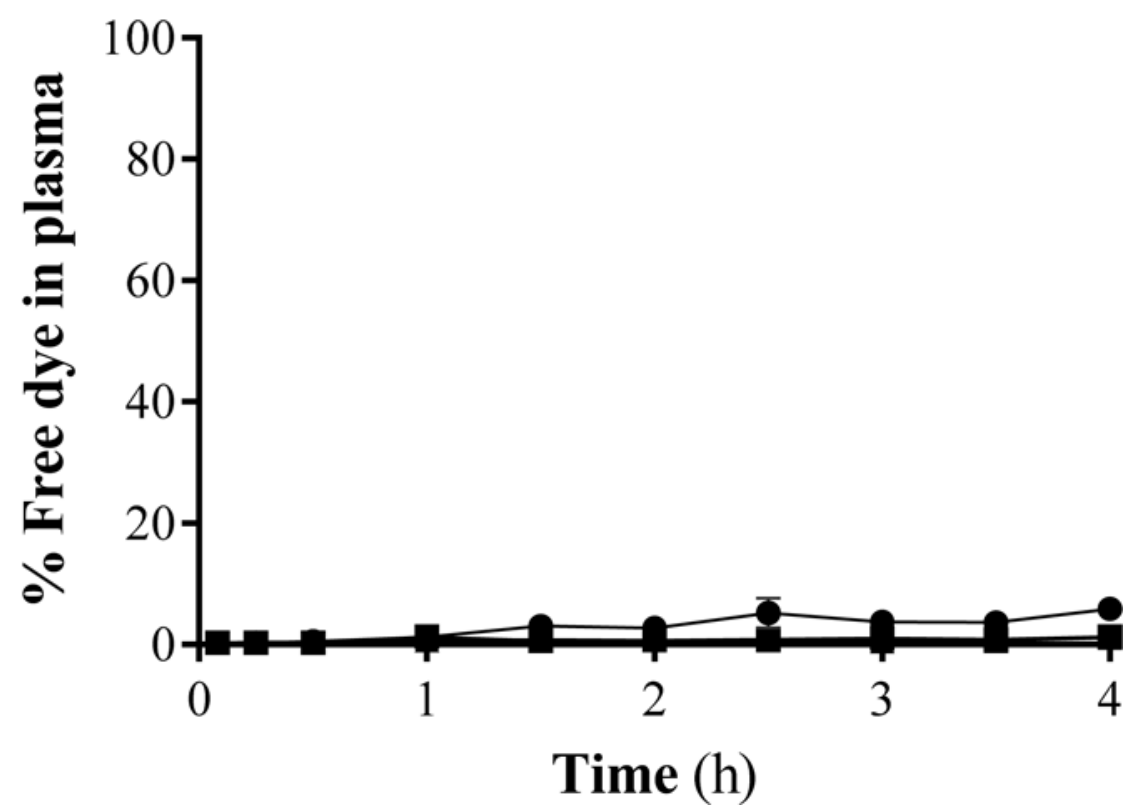

Figure 1. Plasma Pharmacokinetics of ELP Constructs in a Rat Pregnancy Model.

Blood was sampled at various time points up to $4 \mathrm{~h}$ after bolus i.v. injection. A. Plasma levels of ELP proteins. B. Effects of fluorophore loss from polypeptides were assessed by measuring the plasma fluorescence before and after precipitation of the proteins with TCA. Values are mean $\pm \mathrm{SD}, \mathrm{n}=4$, *ELP-63 levels significantly different from ELP-127 and ELP-223, **ELP-127 levels significantly different from ELP-223 as assessed by a two-way repeated measures ANOVA with post hoc Tukey's multiple comparison, $\mathrm{p} \leq 0.05$. 
A
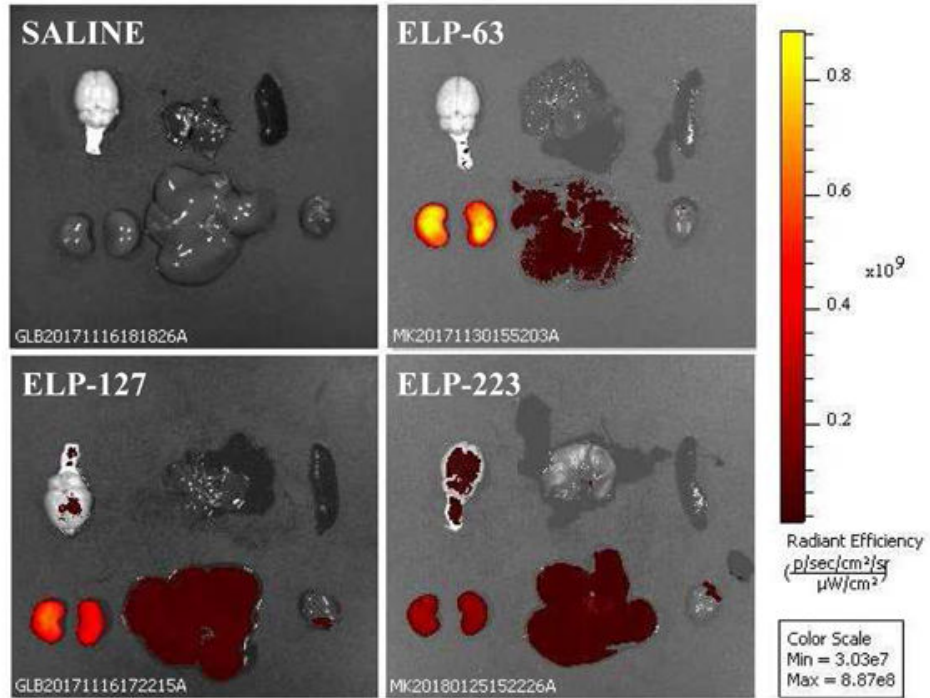

$$
\text { ( } \frac{\mathrm{p} / \mathrm{sec} / \mathrm{cm}^{2} / \mathrm{s}}{\mu \mathrm{W} / \mathrm{cm}^{2}}
$$

B
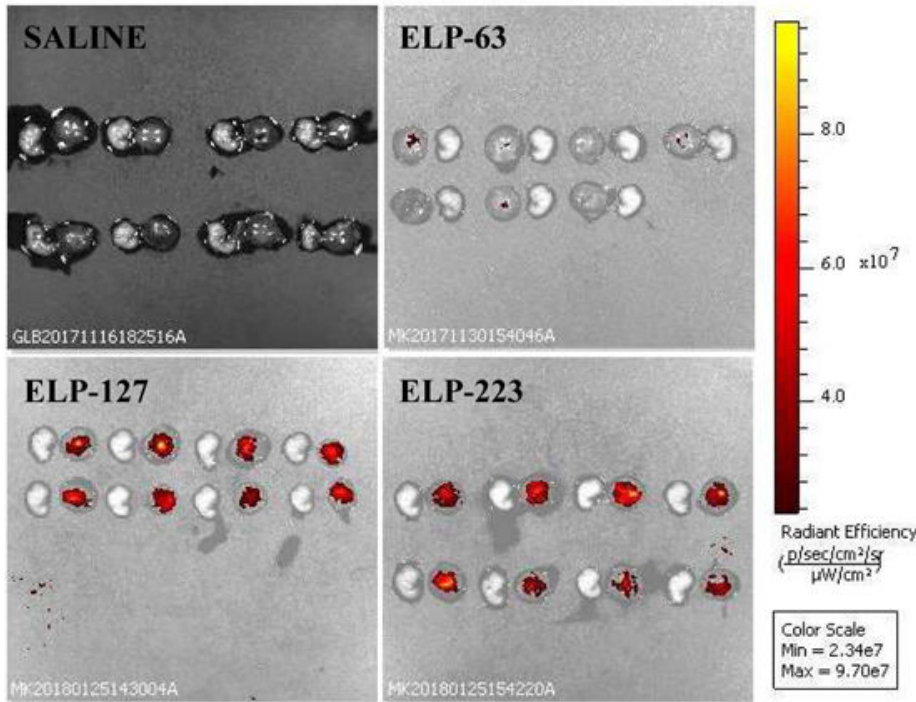

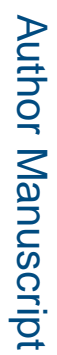




\section{C}

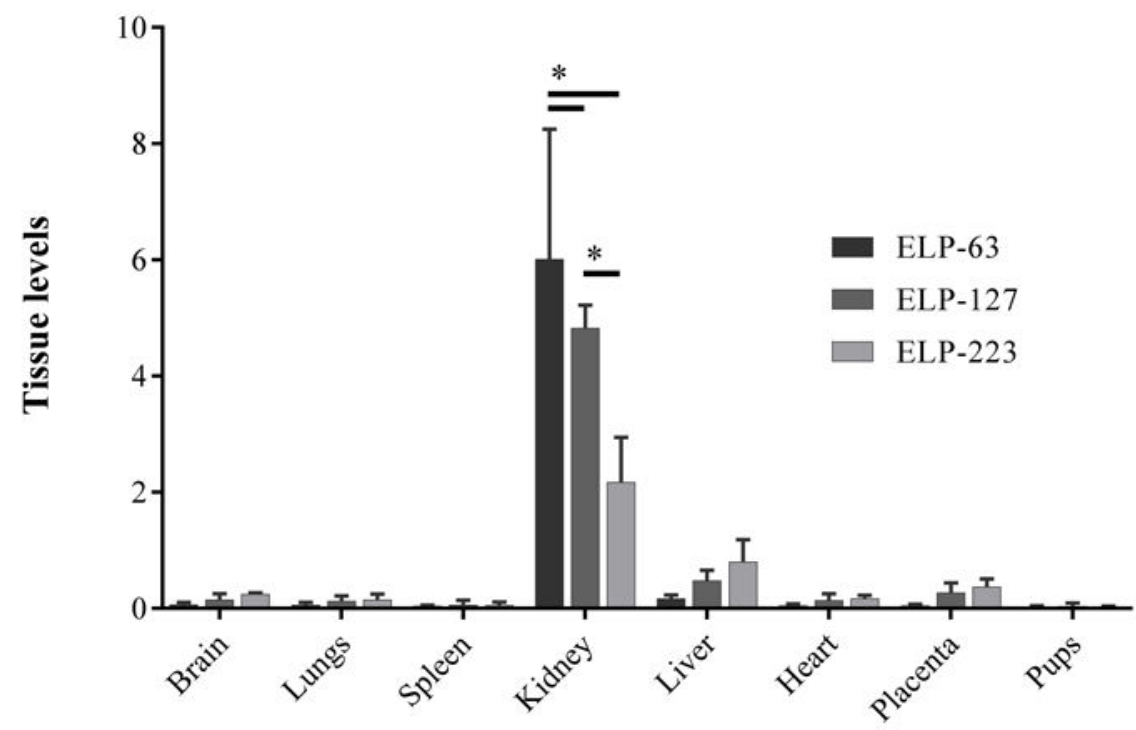

D

$* \mathrm{p} \leq 0.05$
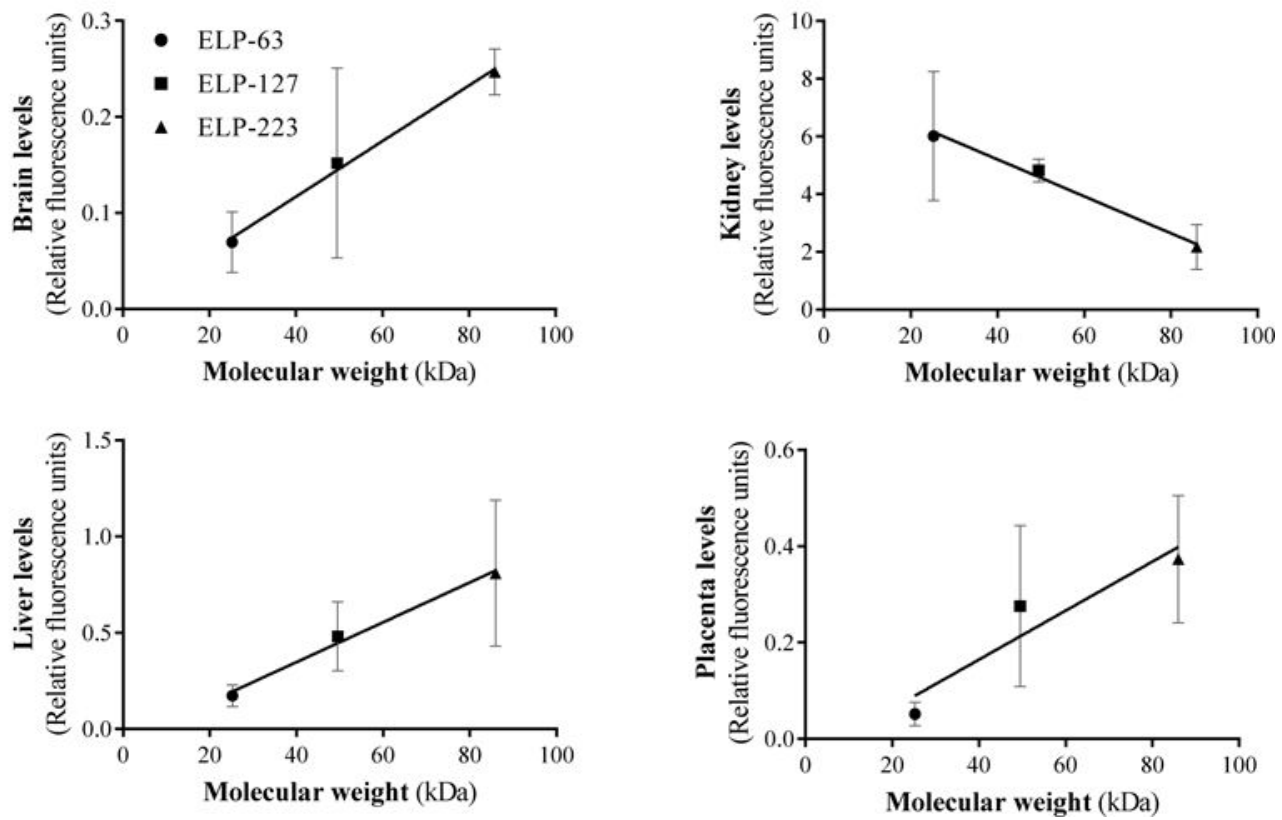

Figure 2. Tissue Biodistribution of ELP Constructs in a Rat Pregnancy Model.

Fluorescence levels in tissue were determined four hours after bolus i.v. injection in placentae, pups and major organs by ex vivo whole organ fluorescence imaging. A. Representative image of major organs from one animal from each group. B. Representative image of placentae and corresponding pups from one animal from each group. C. ELP tissue biodistribution 4 hours after administration. D. ELP levels in major organs as a function of MW were fit by linear regression using GraphPad Prism. Values are mean \pm SD, $n=4$, at 
least five pups per rat. *Statistically significant difference between indicated groups as assessed by a two-way ANOVA with post hoc Tukey's multiple comparison, $\mathrm{p} \leq 0.05$. 
A

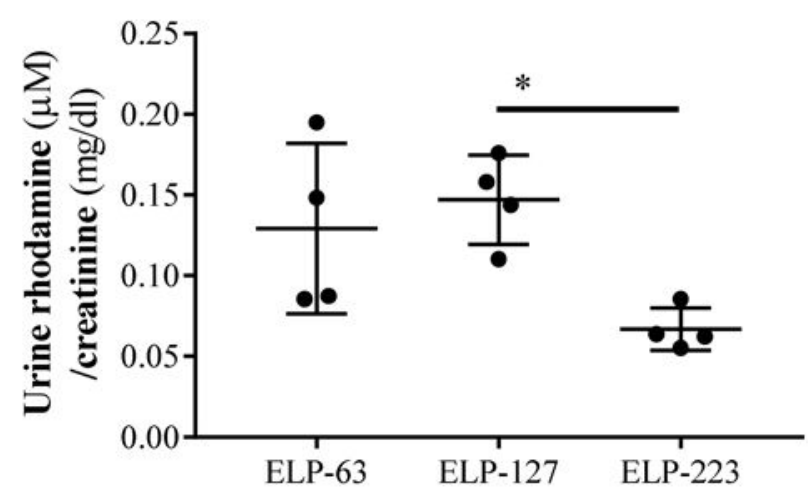

C

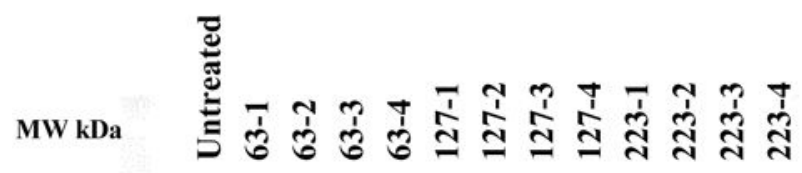

75

25

B

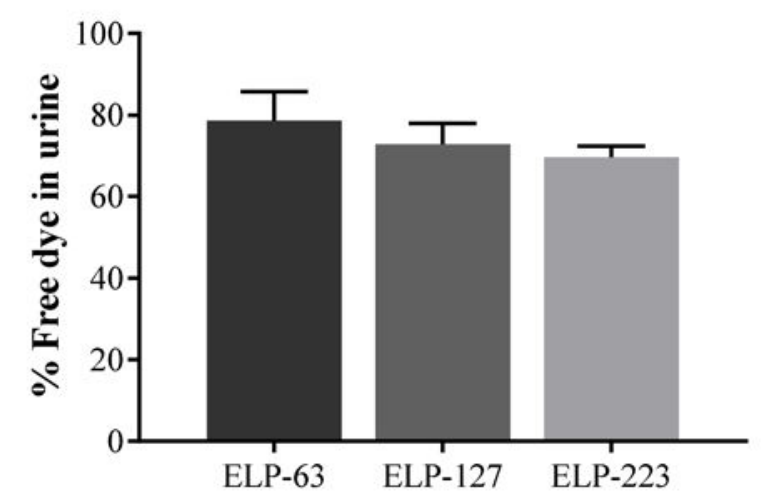

D

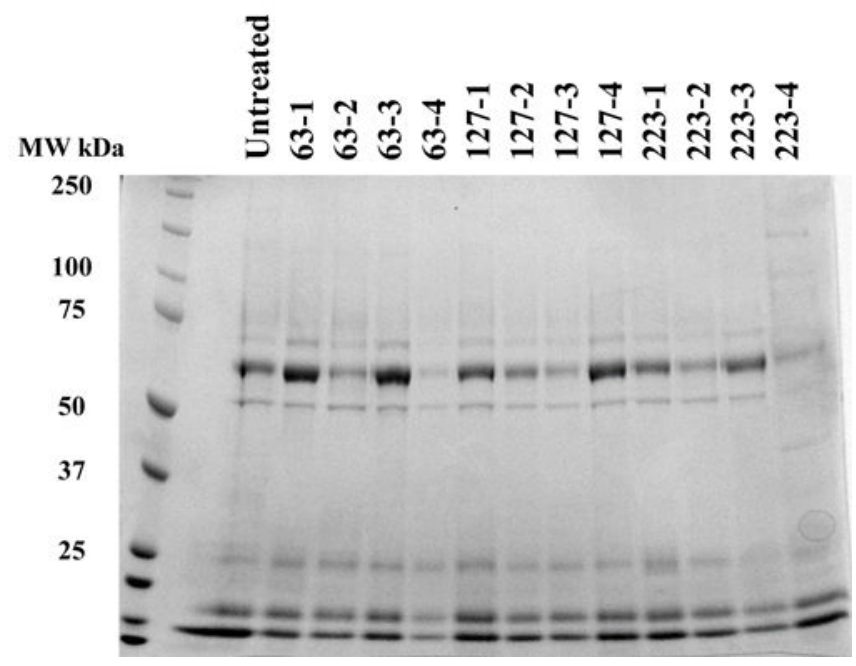

Figure 3. Levels of Rhodamine in the Urine.

Fluorescence levels in urine were determined four hours after bolus i.v. injection by direct fluorescence measurement of urine samples before and after precipitation of the proteins with TCA, and corrected for control. A. Fluorescence was corrected for slight differences in injection volume, fit to a standard curve, and normalized by creatinine level. B. Percentage of the urine signal coming from free fluorophore as assessed after TCA precipitation of the protein-bound fraction. C and D. SDS-PAGE analysis of urine samples with direct fluorescence imaging (C) and Coomassie staining (D). Values are mean $\pm S D, n=4$. *Statistically significant difference between indicated groups as assessed by one-way ANOVA with post hoc Tukey's multiple comparison, $\mathrm{p} \leq 0.05$. 
A

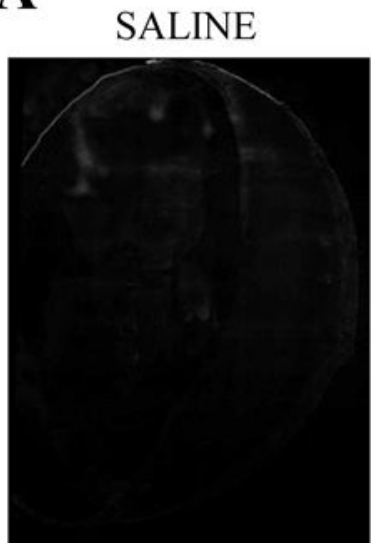

ELP-63

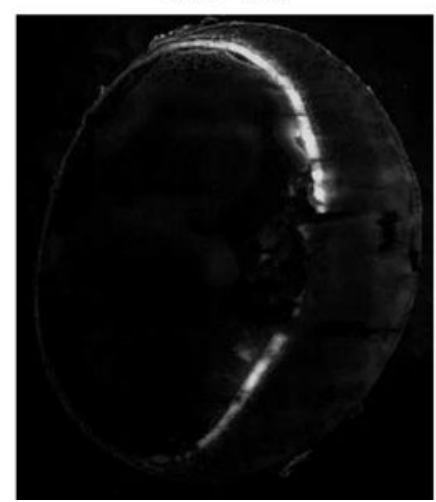

ELP-127

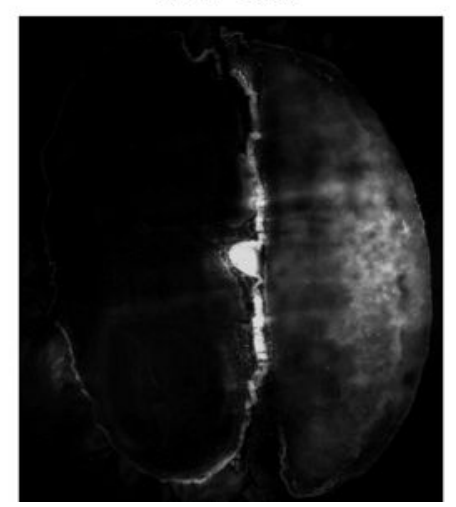

ELP-223

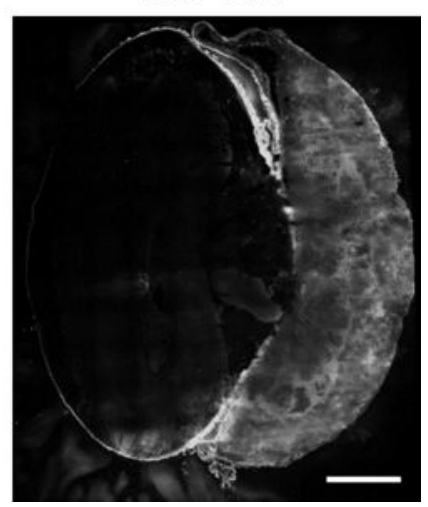

Figure 4. Placental Distribution of ELP Constructs.

A. Placental localization of ELPs in placental sections obtained by confocal microscopy image stitching, scale bar $=2000(\mu \mathrm{m}$. Imaging parameters were identical among all samples, and image intensities represent actual protein levels. 
A

\section{SALINE}

ELP-63

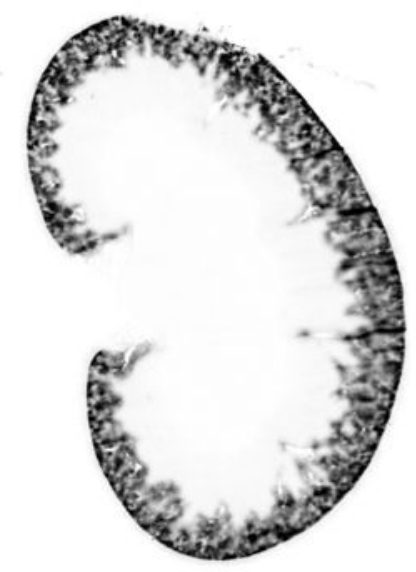

ELP-223

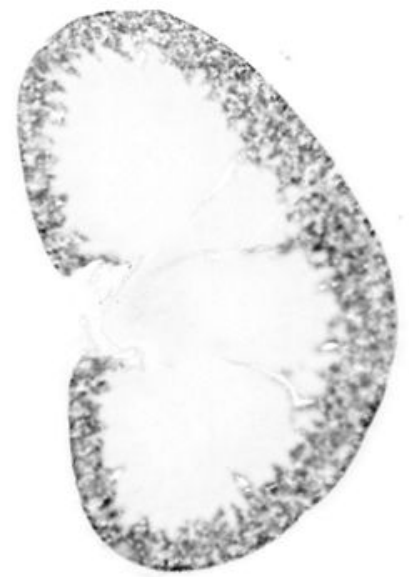

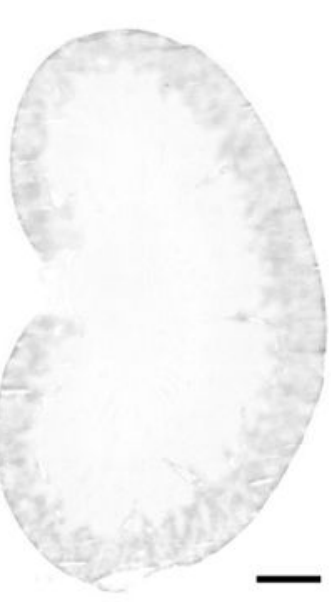

B

ELP-63

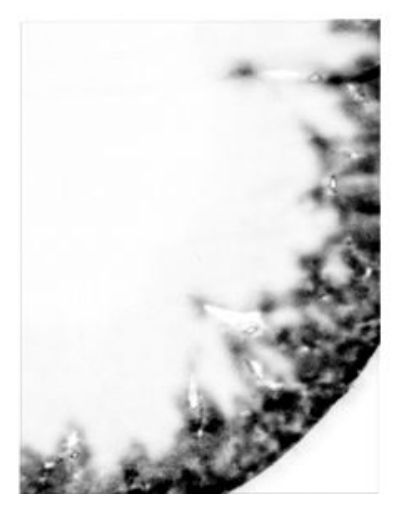

ELP-127

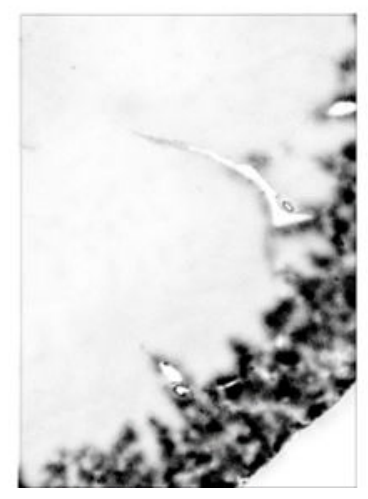

ELP-223

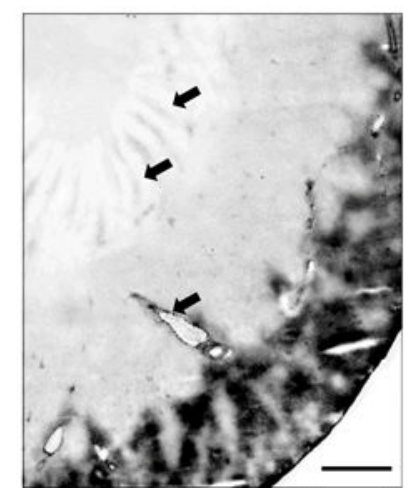

Figure 5. Intrarenal Distribution of ELP Constructs.

A. Intrarenal localization of ELPs in kidney sections obtained by confocal microscopy image stitching using the same settings in order to accurately present protein levels, scale bar $=2000(\mu \mathrm{m}$. B. Magnified view of the kidney with image brightness adjusted to optimize visibility, scale bar $=1000 \mathrm{~nm}$, arrows point to medullary structures. 


\section{Table 1.}

\section{Size and Transition Temperature of the ELP Constructs Used in this Study.}

Predicted molecular weight are based on the primary amino acid sequence, as calculated by the ExPASy ProtParam tool. Transition temperature was measured by turbidity analysis. Hydrodynamic radius was measured by dynamic light scattering ${ }^{*}$.

\begin{tabular}{cccc}
\hline Protein & $\begin{array}{c}\text { Predicted protein } \\
\text { MW (kDa) }\end{array}$ & $\begin{array}{c}\text { Transition } \\
\text { temperature }\left({ }^{\circ} \mathbf{C}\right)\end{array}$ & Radius (nm) \\
\hline ELP-63 & 25.2475 & 89.745 & $4.170 \pm 0.056$ \\
ELP-127 & 49.5469 & 65.775 & $5.800 \pm 0.200$ \\
ELP-223 & 85.996 & 60.250 & $6.830 \pm 0.169$ \\
* & & & \\
Adapted from Kuna, et al., Scientific Reports, 2018 May 21;8(1):7923.
\end{tabular}


Table 2.

Results of Correlation Analysis for MW and Organ Levels.

Reported are Pearson correlation coefficient, $\mathrm{R}^{2}$ and $\mathrm{p}$ value for three $\mathrm{xy}$ pairs.

\begin{tabular}{cccc}
\hline & $\mathbf{r}$ & $\mathbf{R}^{2}$ & P value \\
\hline Brain & 0.9973 & 0.9946 & 0.0468 \\
Lungs & 0.9282 & 0.8615 & 0.2427 \\
Spleen & 0.495 & 0.245 & 0.6703 \\
Kidney & -0.9948 & 0.9897 & 0.0648 \\
Liver & 0.9953 & 0.9907 & 0.0615 \\
Heart & 0.9341 & 0.8726 & 0.2324 \\
Placentae & 0.9436 & 0.8904 & 0.2148 \\
Pups & 0.255 & 0.06503 & 0.8358
\end{tabular}

